\title{
Negative Poisson's Ratio Behavior Induced by an Elastic Instability
}

\section{Citation}

Bertoldi, Katia, Pedro M. Reis, Stephen Willshaw, and Tom Mullin. 2010. Negative Poisson's Ratio Behavior Induced by an Elastic Instability. Advanced Materials 22(3): 361-366.

\section{Published Version}

doi:10.1002/adma.200901956

\section{Permanent link}

http://nrs.harvard.edu/urn-3:HUL.InstRepos:11051225

\section{Terms of Use}

This article was downloaded from Harvard University's DASH repository, and is made available under the terms and conditions applicable to Open Access Policy Articles, as set forth at http:// nrs.harvard.edu/urn-3:HUL.InstRepos:dash.current.terms-of-use\#OAP

\section{Share Your Story}

The Harvard community has made this article openly available.

Please share how this access benefits you. Submit a story.

Accessibility 
DOI: $10.1002 /$ adma.

\section{Novel negative Poisson's ratio behavior induced by an elastic instability}

By Katia Bertoldi*, Pedro M. Reis, Stephen Willshaw and Tom Mullin

[*] K. Bertoldi,

Multiscale Mechanics,

University of Twente,

PO Box 217, 7500 AE Enschede, NL.

E-mail: K.Bertoldi@ctw.utwente.nl

P. M. Reis,

Department of Mathematics, \#2-335,

Massachusetts Institute of Technology,

77 Massachusetts Avenue,

Cambridge, MA 02139-4307, USA.

S. Willshaw, T. Mullin,

Department of Physics \& Astronomy,

University of Manchester,

Manchester, M13 9PL, U.K.

Keywords: (Poisson's ratio, auxetic, instability, experimental, numerical.)

When materials are compressed along a particular axis they are most commonly observed to expand in directions orthogonal to the applied load. The property that characterizes this behavior is the Poisson's ratio which is defined as the ratio between the negative transverse and longitudinal strains. The majority of materials are characterized by a positive Poisson's ratio which is approximately 0.5 for rubber and 0.3 for glass and steel. Materials with a negative Poisson's ratio will contract (expand) in the transverse direction when compressed (stretched) and, although they can exist in principle, demonstration of practical examples is relatively recent. Discovery and development of materials with negative Poisson's ratio, also called auxetics, was first reported in the seminal work of Lakes in $1987 . .^{[1]}$

There is significant interest in the development of auxetic materials because of tremendous potential in applications in areas such the design of novel fasteners ${ }^{[2]}$, prostheses ${ }^{[3]}$, piezocomposites with optimal performance ${ }^{[4]}$ and foams with superior damping and acoustic properties ${ }^{[5]}$. The results of many investigations ${ }^{[6-7]}$ suggest that the auxetic behavior involves an interplay between the microstructure of the material and its deformation. Examples of this are provided by the discovery that metals with a cubic lattice ${ }^{[8]}$, natural layered ceramics ${ }^{[9]}$, ferroelectric polycrystalline ceramics ${ }^{[10]}$ and zeolites ${ }^{[11]}$ may all exhibit negative Poisson's ratio behavior. Moreover, several geometries and mechanisms have been proposed to achieve negative values for the Poisson's ratio, including foams with reentrant structures ${ }^{[1]}$, hierarchical laminates ${ }^{[12]}$, polymeric and metallic foams ${ }^{[13]}$, microporous polymers ${ }^{[14]}$, molecular networks ${ }^{[15]}$ and manybody systems with isotropic pair interactions ${ }^{[16]}$. Negative Poisson's ratio effects have also been demonstrated at the micron scale using complex materials which were fabricated using soft lithography ${ }^{[17]}$ and at the nanoscale with sheets assemblies of carbon nanotubes ${ }^{[18]}$.

A significant challenge in the fabrication of materials with auxetic properties is that it usually involves embedding structures with intricate geometries within a host matrix. As such, the manufacturing process has 
been a bottleneck in the practical development towards applications. A structure which forms the basis of many auxetic materials is that of a cellular solid and research into the deformation these materials is a relatively mature field ${ }^{[19]}$ with primary emphasis on the role of buckling phenomena on load carrying capacity and energy absorption under compressive loading. Very recently, the results of a combined experimental and numerical investigation demonstrated that mechanical instabilities in 2D periodic porous structures can trigger dramatic transformations of the original geometry ${ }^{[20,21]}$. Specifically, uniaxial loading of a square array of circular holes in an elastomeric matrix is found to lead to a pattern of alternating mutually orthogonal ellipses. This results from an elastic instability above a critical value of the applied strain. The geometric reorganization observed at the instability is both reversible and repeatable and it occurs over a narrow range of the applied load. Thus, this behavior provides opportunities for transformative materials with properties that can be reversibly switched. Similar instability induced pattern transformations have been observed also at the sub-micron scale ${ }^{[22,23]}$. These observations pave the way for the development of a new class of materials which take advantage of such behavior.

Here we exploit elastic instabilities to create novel effects within materials with periodic microstructure. We show that the pattern transformation leads to unidirectional negative Poisson's ratio behavior for the 2D structure i.e. it only occurs under compression. The uncomplicated manufacturing process of the samples together with the robustness of the observed phenomena suggests that this may form the basis of a practical method for constructing planar auxetic materials over a wide range of length-scales. Excellent quantitative agreement is found between numerical and experimental results to illustrate the effect for a specific sample. The numerical approach is subsequently used to explore the effect of void fraction and we uncover a scaling law for the phenomenon. Finally, we draw some conclusions and give future perspectives for this simple yet novel auxetic material.

Our system comprised a square lattice of circular holes in an elastomeric matrix which was subjected to uniaxial compression using an Instron machine as described in the Experimental Details Section. A representative sequence of images of the sample during loading is presented in Figure 1 where the image shown in Figure 1a corresponds to the undeformed sample, prior to loading. During the initial response of the periodic structure, the circular holes were observed to undergo a gradual and homogeneous compression and this corresponds to a linearly elastic regime at low strains ${ }^{[20]}$. As will be discussed in detail below, above a critical value of compressive nominal strain of $\epsilon_{\mathfrak{c}}=-0.047$ a transformation to a pattern of alternating, mutually orthogonal ellipses was observed and an example of the resulting pattern switch is shown in Figure $1 \mathrm{~b}$ where the image was taken at $\epsilon=-0.06$ nominal strain. Once formed, the new pattern became further accentuated with increasing macroscopic compressive strain as may be seen in the image shown in Figure 1c taken at $\epsilon=-0.25$ nominal strain. A feature which is clear in Figure 1c is that the lateral boundaries of the sample bend inwards, a clear signature of negative Poisson ratio behavior.

The experiments were performed on a silicone rubber cellular solid, which was manufactured using the molding process described in the Experimental Details Section. The deformation of the sample was monitored using a high-resolution digital camera (6 MPixels Nikon D100 SLR) and which was synchronized with the Instron. Quantitative estimates of the deformation of the holes in the sample were made using purpose made image processing software. In outline, at a particular value of strain, the $\left(x_{i, j}, y_{i, j}\right)$ coordinates of the centroid of each void were determined where $1<i<10$ and $l<j<10$ are the row and column indices, respectively. Hence a particular index pair $(i, j)$ is unique to each specific hole. This part of the procedure was accomplished using an edge detection algorithm (Matlab: Image Processing Toolbox), which takes advantage of the high contrast between the bulk regions in the sample and the voids. It became clear that the deformation near the four edges of the specimen was strongly influenced by the boundary conditions and 
hence, we focused on the behavior of the central part of the sample where the response was clearly more uniform. Thus we tracked the centroids of the sixteen central voids (delimited by the dashed rectangle in Figure $1 \mathrm{~b}$ for holes with indices $4 \leq i \leq 7$ and $4 \leq j \leq 7$ ). The horizontal and vertical controid-to-centroid distances were calculated from the coordinates $\left(x_{i, j}, y_{i, j}\right)$ i.e. $\Delta x_{i, j}=x_{i+1, j^{-}} x_{i, j}$ and $\Delta y_{i, j}=y_{i, j+1^{-}} y_{i, j}$, respectively. Prior to compression, the value for both of these quantities was the center-to-center distance between the undeformed circular holes i.e. $\Delta x(0)=\Delta y(0)=11.95 \mathrm{~mm}$. A schematic diagram of the central region under consideration illustrating the definitions of $\Delta x_{i, j}$ and $\Delta y_{i, j}$ shown in Figure 1d.

Numerical investigations were performed on both finite-sized and periodic domains using the nonlinear finite element code ABAQUS. Details of the method are provided elsewhere ${ }^{[21]}$ and here we highlight some of the parts of the numerical procedure which are specific to the current investigation. Each mesh was constructed using six-node, quadratic, hybrid, plane strain elements (ABAQUS element type CPE6H) and the accuracy was checked by mesh refinement. The material was modeled using a two-term I1-based Rivlin model, which was modified to include compressibility with a high bulk modulus ${ }^{[21]}$ where the material properties were determined by fitting the model to experimental calibration data (see Experimental Details Section). The instabilities were initially investigated using a linear perturbation procedure and the results confirmed that the observed pattern transformation arose from an elastic instability, since it corresponds to the lowest eigenmode of the structure. An imperfection was introduced into the mesh in the form of this eigenmode which enabled load-displacement analysis for the finite-sized structures. This procedure captured the instability induced pattern transformation and boundary effects were included so that direct comparison could be made with experiment. Calculations were also performed on infinite periodic structures and were used primarily for parametric investigations of the phenomena since carrying out a similar experimental study would have been impractical.

In Figure $2 \mathrm{a}$ we present the functional dependence of both $\left.<\Delta x_{i, j}\right\rangle / \Delta x(0)$ and $\left.<\Delta y_{i, j}\right\rangle / \Delta y(0)$ on nominal strain, where the angular brackets $<.>$ denote ensemble average over the sixteen holes (hence, nine values of $\Delta x_{i, j}$ and $\left.\Delta y_{i, j}\right)$ under consideration $(4 \leq i<7$ and $4 \leq j<7)$. The experimental data points are shown in comparison with the numerically determined solid lines and it can be seen that there is close agreement between the sets of results. The normalized vertical centroid-to-centroid distance shows a monotonic decrease from one (undeformed case) to approximately 0.85 at a strain of $\epsilon=-0.1$. On the other hand, the horizontal centroidto-centroid distance exhibits a non-monotonic dependence on nominal strain. It first increases during the initial linear elastic response of the periodic structures and, once the pattern switch occurs $(\epsilon=-0.047)$, it decreases.

The center-to-center distances of the holes were used to calculate local values of the Poisson's ratio using

$$
v_{i, j}=-\frac{\Delta x_{i, j} / \Delta x(0)}{\Delta y_{i, j} / \Delta y(0)} .
$$

The ensemble average $v=\left\langle v_{i, j}\right\rangle$ for the sixteen central voids under consideration was computed. The experimental and numerical estimates of $v$ are plotted as a function of nominal strain in Figure $2 \mathrm{~b}$ where the error bars on the experimental points were obtained from the standard deviation of the nine values of $v_{i, j}$ used in the averaging. When $\varepsilon<-0.047$, the response of the sample is linear as in the stress strain plot in Figure 2c. Over this range of strain, the numerically determined estimate of the Poisson's ratio is approximately constant at $v=0.284 \pm 0.014$. The experimental data on the other hand lies consistently below the numerical results at a value of $v=0.221 \pm 0.021$ and shows a slight increase. Over this range of strain, the deformation of the holes is very small and hence so are $\Delta x_{i, j}$ and $\Delta y_{i, j}$. Therefore accurate measurements are difficult to make since small unavoidable alignment errors between the sample and the camera will dominate. 
It can be seen in Figure 2c that a plateau emerges in the stress-strain curve for strains in excess of $\varepsilon_{c}=-0.047$ i.e. the total stress becomes independent of strain. This type of response is generic for cellular solids under compression $^{[19]}$ and the initial periodic structure is recovered upon removal of the strain so that the change is reversible and repeatable. The strain $\varepsilon_{c}=-0.047$ corresponds to the critical strain at which the pattern transformation occurs and we have indicated this by a vertical line which runs through all parts of Figure 2. The Poisson's ratio starts to decrease above this point and eventually becomes negative for strains in excess of $\varepsilon=-0.053 \pm 0.003$. Interestingly, the agreement between the experimental and numerical results for estimates of the Poisson's ratio shown in Figure 2b, improves considerably once the pattern transformation occurs and there is an obvious distortion of the holes as shown in Figure 1b. This excellent agreement between the two sets of results can be seen for further increase in strain and the minimum value of the Poisson's ratio found in the experiments was $v=-0.538$ for $\varepsilon=-0.10$. Beyond this value, the top and bottom edge of some voids touched and determination of the position of the centroids was problematic, although further contraction in the transverse direction is evident as shown in Figure 1c.

The results reported thus far are for a particular value of the void fraction, $\Phi=0.5$, where $\Phi$ is defined as the ratio of the total area of voids divided by the surface area of the sample. The finite element simulations are able to accurately reproduce the experimental results and we now perform numerical investigations of the dependence of the auxetic properties on $\Phi$. The pattern switch which underpins the auxetic behavior occurs throughout the sample and is only marginally influenced by the boundaries. Moreover, practical applications are likely to involve samples of a large spatial extent. Consideration of both of these points, directed our focus to considering infinite periodic structures using Representative Volume Elements (RVEs) and numerically convenient periodic boundary conditions ${ }^{[21]}$. Two different types of instability may occur ${ }^{[21]}$ in infinite periodic solids under compression on periodic domains: microscopic instabilities with a finite wavelength and macroscopic instabilities characterized by a wavelength much larger than the scale of the microstructure. Although microscopic instabilities alter the original periodicity of the solid, here they are investigated on the primitive cell using Bloch wave analysis ${ }^{[21]}$ which provides both the point on loading path where instability occurs and the periodicity of the new structure. Macroscopic instability is also detected by monitoring the loss of ellipticity of the homogenized tangent modulus ${ }^{[21]}$.

Both microscopic and macroscopic instabilities were investigated and the effect of the void volume fraction on the response was studied. The results reported in Figure $3 \mathrm{a}$ show that for $\Phi<0.34$, a macroscopic instability is the first to occur along the loading path. This leads to localized deformation in the form of a collapsed band of holes normal to the direction of the applied load as shown schematically in the top inset in Figure 3a. By way of contrast, a microscopic instability was found to occur first for $\Phi>0.34$. Here the mode of deformation is characterized by a critical eigenmode consisting of mutually orthogonal ellipses as shown in the inset in the lower left hand corner of Figure $3 \mathrm{a}$ and consistent with the experiments. As for the finitesized sample, load-displacement analysis was performed for the infinite periodic structures to capture the evolution of the Poisson's ratio during deformation. The Poisson's ratio was calculated directly form the numerical results on the RVEs using the homogenized strains in axial and lateral directions. As a check, it was also estimated from the images of the calculated patterns using the distance between the centers of the voids as in the experiments and negligible difference between the results obtained using the methods was found.

Results for the evolution of the Poisson's ratio as a function of the applied nominal strain are presented in Figure $3 \mathrm{~b}$ for various values of void volume fraction and a strong dependency on this parameter is evident. For $\Phi<0.34$ (where localization occurs prior to the pattern transformation) the Poisson's ratio is always 
positive during loading. On the other hand, samples with $\Phi>0.34$ are characterized by behavior analogous to the experimental samples discussed above viz. positive Poisson's ratio up to a critical value of strain, $\varepsilon_{c}$, at which the instability induced pattern transformation occurs and beyond which the Poisson ratio decreases until it eventually becomes negative. It is interesting to note that the lowest value of the asymptotic Poisson's ratio we were able to reach numerically was $v_{\infty}=-0.904$, which occurs for the highest value of void fraction investigated $\Phi=0.70$.

It is striking that simply by increasing the void fraction, samples can be constructed to attain increasingly negative values of $v$ in a pronounced way. In particular, the three quantities of interest - the value of strain at which the pattern transformation occurs, $\varepsilon_{c}$, the asymptotic value of the Poisson ratio and the rate at which this asymptotic value is reached are - all strongly depend on void fraction. We now explore the dependence of these quantities on void fraction in detail. In Figure 4 a we show the Poisson's ratio dependence on $\varepsilon-\varepsilon_{\mathrm{c}}$ for a range of void fractions. We see that the results from the RVE simulations (circles) can be accurately fitted (solid lines) by exponentials of the form

$$
v=v_{\infty}+\left(v_{c}-v_{\infty}\right) \exp \left[-\frac{\varepsilon-\varepsilon_{c}}{\varepsilon_{o}}\right]
$$

where, $v_{\infty}$, is the asymptotic Poisson's ratio, $v_{c}$ is the Poisson's ratio at the onset of the instability which occurs at a nominal strain, $\varepsilon_{\mathrm{c}}$, and the characteristic strain of decay, $\varepsilon_{0}$, measures the speed the asymptotic value is reached.

The exponential fits are particularly good when $\Phi<0.56$. Small deviations are observed for $\Phi>0.56$ but the exponential dependence of Equation (2) is nonetheless a satisfactory way to describe the data from the RVE simulations. In Figure $4 \mathrm{~b}$, we plot the dependence of the fitting parameters $v_{\infty}$ and $\varepsilon_{0}$ on void fraction and the error bars were obtained from the statistical errors in the fitting procedure. The asymptotic value of the Poisson's ratio, $v_{\infty}$, is negative for all void fractions when $\Phi>0.34$ i.e. where there was an instability induced pattern transformation. Moreover, $v_{\infty}$ exhibits a monotonic decrease with two approximately linear regimes separated by a kink at $\Phi \sim 0.46$. On the other hand, the characteristic strain of decay, $\varepsilon_{0}$, first increases to a maximum at $\Phi \sim 0.46$, beyond which it decays. This is significant of a larger rate of decay $\left(1 / \varepsilon_{0}\right)$ of $v$ for high void fractions. These results suggest that in order to maximize auxetic properties, samples should be manufactured with the largest possible void fraction.

We have shown that a cellular solid which comprises a solid matrix with a square array of voids displays two-dimensional negative Poisson's ratio behavior. Unlike many other examples of auxetic materials the effect is only found under compression and above a critical value of an applied strain. The behavior arises as a result of a pattern transformation which has recently found application at the micron scale in phononic/photonic crystals ${ }^{[24,25]}$. The insight gained by performing a numerical parametric exploration serves as an important design guideline in fabricating practical materials towards applications. The overriding features of the system we have explored are the simplicity of the construction and the robustness of the behavior. Each of these aspects points to a more practical method for producing negative Poisson's ratio material which can operate over a wide range of scales.

Experimental Details.

\section{Material}

Experiments were performed on samples made from the addition curing silicone rubber "Sil AD Spezial" (SADS), manufactured by "Feguramed GmbH". The manufacture involved mixing equal measures of two 
fluids, placing the individual component fluids under vacuum to remove dissolved gases and allowing the mixture to set for an hour to ensure proper curing.

Uniaxial compression and tension stress-strain tests were conducted to characterize the behavior of the bulk material by measuring stress/strain relationships for specifically designed solid blocks using a standard Instron apparatus with a $100 \mathrm{kN}$ load cell. We found that the elastomeric stress-strain behavior is well captured using an incompressible two-term I1-based Rivlin model ${ }^{[21]}$, so that the nominal stress is given by

$$
s=\left(\lambda-\frac{1}{\lambda^{2}}\right)\left[\frac{\mu}{2}+4 c\left(\lambda^{2}+\frac{2}{\lambda}-3\right)\right],
$$

where $\mu$ is the initial shear modulus and $\lambda$ denotes the applied stretch is $\lambda=\varepsilon+1$. The required material parameters were found by fitting Eqn. (3) to the experimental data obtained from the calibration tests. A Young's modulus of $470.8 \pm 1.6 \mathrm{kPa}$ was determined so that $\mu=156.9 \pm 0.5 \mathrm{kPa}$ and using c$=20.1 \pm 0.4 \mathrm{kPa}$ the material behavior was found to be well captured up to a nominal strain of 0.6 both in tension and in compression.

\section{Rectangular Sample Manufacture}

A batch of SADS mixture was poured into an aluminum mould measuring 130 x 130 x 7.9 mm. Machined brass pins of diameter $10 \mathrm{~mm}$ were screwed into the base of the mould to create a square array of 100 holes with a center to center spacing of $12.0 \mathrm{~mm}$. The mould was coated with a thin layer of vaseline to help prevent sticking of the rubber to the metal. The mould was sealed and the mixture was allowed to set at atmospheric pressure and room temperature. The sample was removed from the mould after one hour and two of the side walls were cut from the sample, leaving eight columns of ten holes, flanked by a column of ten semi circles on either side. It was found that a small amount of shrinkage had taken place. The rectangular sample used in the experiments measured 128.7 x $107.0 \times 7.9 \mathrm{~mm}$, with a hole diameter 9.95+ $0.02 \mathrm{~mm}$. Sample dimensions were measured using digital calipers.

\section{Compression Experiments}

Compression tests were performed using an "Instron 5569" machine with a $100 \mathrm{kN}$ load cell. The sample was held vertically between two $8.1 \mathrm{~mm}$ thick PMMA sheets which were held $9.4 \mathrm{~mm}$ apart in the machined slots on a metal base. The sample faces were dusted with flour to reduce any frictional effects resulting from buckling during the loading process. A PMMA sheet of thickness $8.1 \mathrm{~mm}$ was mounted on the lower face of the load cell and used to apply the load to the top of the sample. It was ensured that the three PMMA sheets were parallel to one another, and that the bottom of the loader was parallel to the top of the sample. This allowed the even loading of the sample and prevented the loader touching the outer faces of the housing.

The compression tests were performed by lowering the loader to rest on to the top surface of the sample. The loader was then lowered further, at a rate of approximately $0.01667 \mathrm{~mm}$ per sec, to a final displacement of $30 \mathrm{~mm}$. The results were independent of the rate of change of displacement for slower speeds and hence a good approximation to the rate independent conditions of the numerical investigation was obtained in the experiments. The load associated with the displacement was recorded once per every 100 msecs. and used to produce a stress-strain curve for the compression process. 
Manchester for use of their Instron and helpful discussions. KB and TM are grateful to S. Luding for the helpful discussions and traveling support.

[1] Lakes, R.S. Science, 235,1038, 1987.

Received: ((will be filled in by the editorial staff))

Revised: ((will be filled in by the editorial staff)) Published online: ((will be filled in by the editorial staff))

[2] Choi, J. B. and Lakes, R. S., Cell. Pol., 10, 205, 1991.

[3] Scarpa, F . IEEE Sig. Proc. Mag., 25, 128, 2008.

[4] Sigmund O, Torquato S and Aksay I.A. J. Mat. Res., 13, 1038, 1998.

[5] Scarpa, F., Ciffo L., and Yates J. Smart Mat. Struc., 13,49, 2004.

[6] Evans K. E. and Alderson A. Adv. Mat., 126,17, 2000.

[7] Lakes R.S. J. Mat. Sci., 26, 2287, 1991.

[8] Baughman, R. H., Shacklette, J. M., Zakhidov, A. A. and Stafstrom, S. Nature, 392, 362, 1998.

[9] Song, F., Zhou, J., Xu, X.,Xu, Y. and Bai, Y. Phys. Rev. Lett., 245502, 2008.

[10] Tan, X., Jo, W., Granzow T., Frederick J., Aulbach E. and Rödel J. Appl. Phys. Lett. 94, 042909,2009.

[11] Grima J.N., Jackson R., Alderson A. and Evans K.E.. Adv. Mat., 12, 1912, 2000.

[12] Milton, G., J. Mech. Phys. Sol., 40, 1105, 1992.

[13] Friis, E. A., Lakes, R. S., and Park, J. B., J. Mat. Sci., 23, 4406, 1988.

[14] Caddock B.D. and Evans K.E., J. Phys. D: Appl. Phys. 22 1877, 1998.

[15] Evans, K. E., Nkansah, M. K., Hutchison, I. J. and Rogers S. C. Nature, 353,124, 1991.

[16] Rechtsman, M.C., Stillinger, F.H. and Torquato S. Phys Rev. Lett. 101, 085501, 2008.

[17] Xu, B., Arias, F., Brittain, S. T., Zhao, X-M., Grzybowski, B., Torquato, S. and Whitesides G.M.. Adv. Mat., 11,1186, 1999.

[18] Hall, L.J., Coluci, V. B., Galvao, D. S., Kozlov, M. E., Zhang,,V S., Dantas O. and Baughman R. H. Science 320,504, 2008.

[19] L.J. Gibson and M. F. Ashby Cellular Solids: Structure and properties. \{Cambridge University Press 1999.

[20] Mullin, T., Deschanel, S. Bertoldi, K. and Boyce M. C. Phys. Rev. Lett. 99, 084301, 2007.

[21] Bertoldi, K., Boyce, M.C., Deschanel, S., Prange, S.M. and Mullin T. J. Mech. Phys. Sol., 56, 2642, 2008

[22] Zhang, Y., Matsumoto, E. A., Peter, A., Lin, P.C., Kamien, R. D. and Yang. S. Nano letters, 8, 1192$1196,2008$.

[23] Jang, J.H., Koh, C.Y., Bertoldi, K., Boyce, M.C. , Thomas, E.L. , Nano letters, 9, 2113, 2009

[24] Singamaneni' S., Bertoldi, K., Chang, S.,Jang, J., Thomas, E.L.,Boyce M.C. and Tsukruk V.V. ACS

Appl. Mater. Interf., 1, 42,2009

[25] Zhu, X.L., Zhang, Y., Chandra, D., Cheng, S.C., Kikkawa, J.M., Yang, S., Applied Physics Letters, 93, 161911,2008 

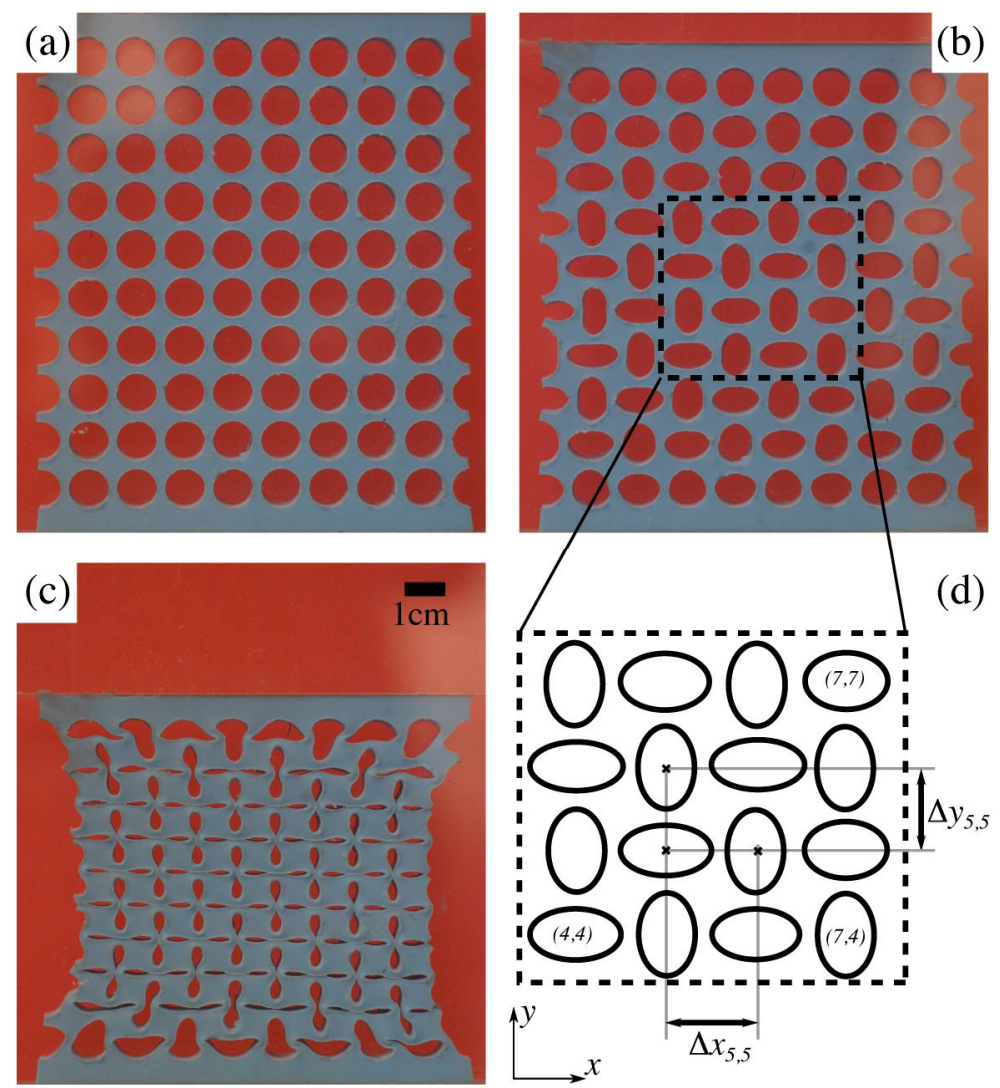

Figure 1. a) Sample in the initial unstrained configuration; b) Sample under compression of $\varepsilon^{=-0.06}$. The dashed rectangle represents 16-hole region over which we perform the ensemble averaging. c) Sample under compression of $\varepsilon=-0.25$. d) Schematic diagram of the central region with 16 holes. The position of the centroids of the holes is measured from image analysis which allows for the determination of the vertical and horizontal center-to-center distance between two consecutive holes. 

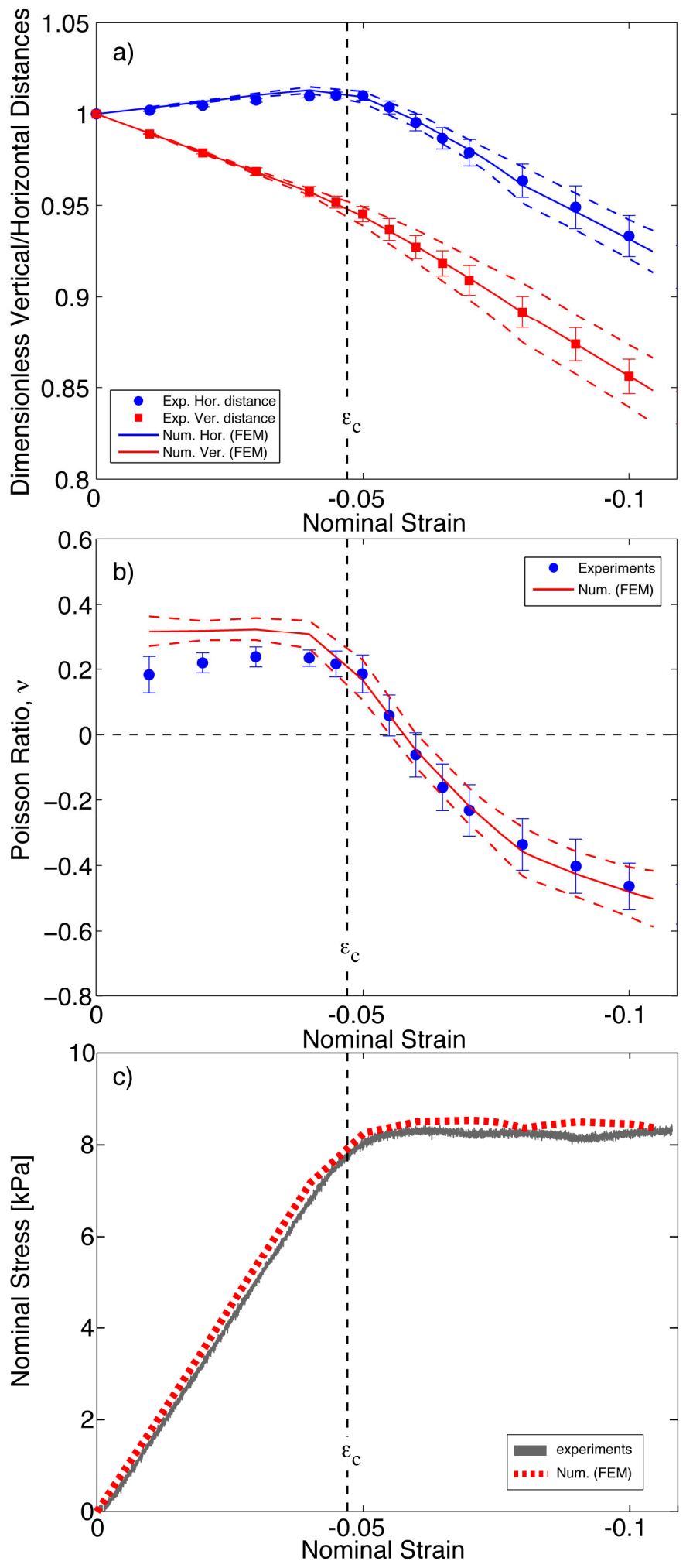

Figure 2. Quantitative comparison between experimental and numerical results a) Averaged dimensionless horizontal and vertical distances of central 16 holes plotted as a function of the applied axial nominal strain b) Poisson's ratio as a function of the applied axial nominal strain and c) Stress-strain response of the experimental sample (solid lines) and results from the finite element calculations (dashed line). In (a) and (b), points correspond to experimental data and lines to the results of the finite element calculations. The solid and dashed lines correspond to the ensemble average and standard deviation, respectively, of the 16 central holes considered (see text for details). 

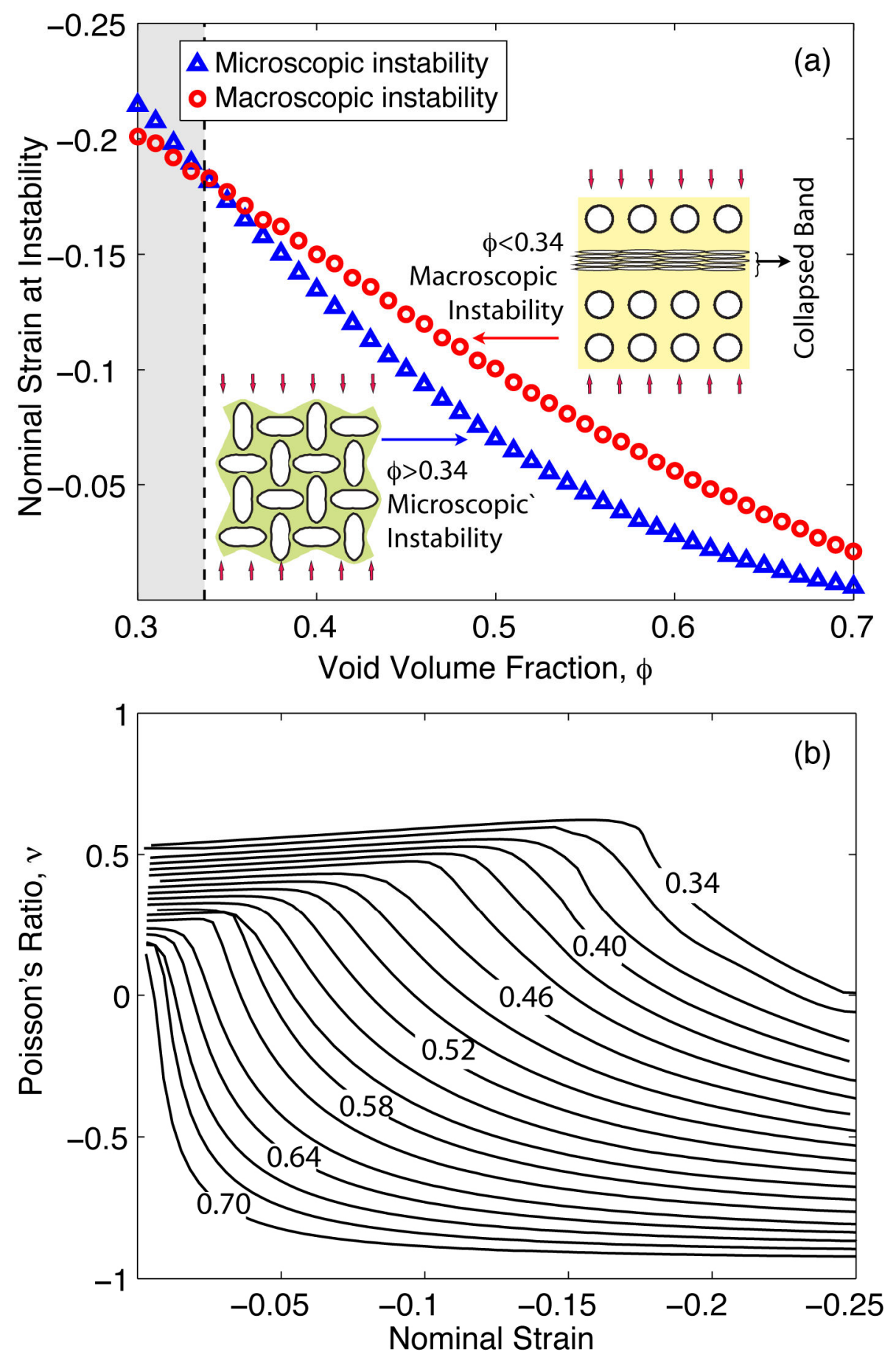

Figure 3. Results of the numerical investigation on the effect of the void volume fraction for an infinite periodic square array of circular holes in an elastomeric matrix a) Nominal strain at the onset instability as a function of the void volume fraction. Inset schematics illustrate the instability type for $\Phi<0.34$ (upper right: localization) and $\Phi>0.34$ (lower left: pattern switch) b) Evolution of the Poisson's ratio as a function of applied axial nominal strain over the range of volume fraction investigated. 

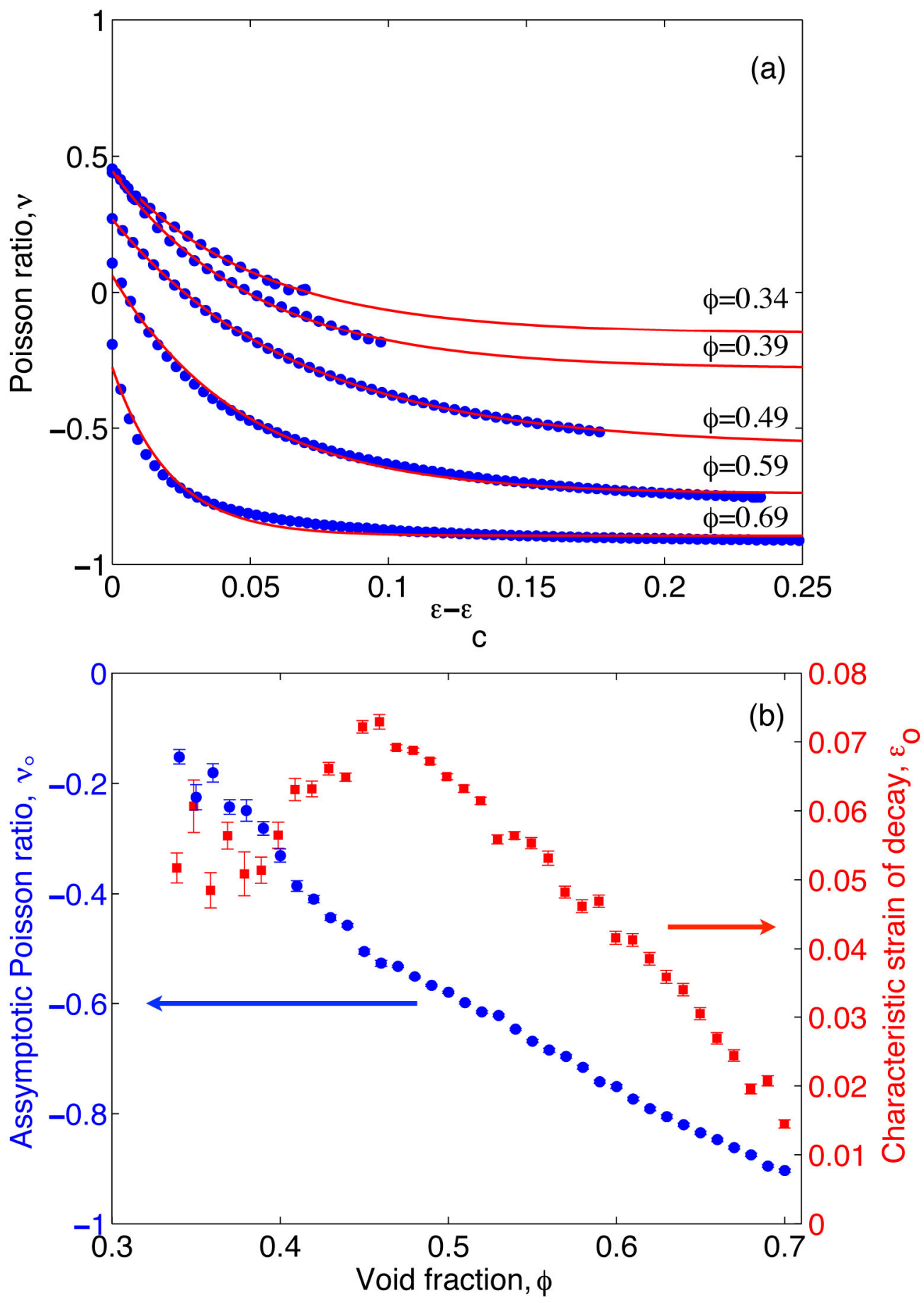

Figure 4.: RVE simulations a) Dependence of the Poisson's ratio on $\varepsilon-\varepsilon_{c}$ (nominal strain above the instability), where $\varepsilon_{c}$ was obtained from the Bloch analysis (plotted in Figure 3a.). The circles correspond to the RVE simulations and the red lines are exponentials. Each set of curves for different void fraction $(0.34$, $0.39,0.49,0.59,0.69)$. b) Parameters obtained from the exponential fit as a function of void fraction: blue circles (left vertical axis) for the asymptotic value of Poisson's ratio, $v_{\infty}$, and red squares (right vertical axis) for strain rate decay, $\varepsilon_{0}$. The red lines are exponentials obtained from fitting the experimental data to Equation (2). 